\title{
Deformation-Controlled Design of Metallic Nanocomposites
}

Hakan Yavas a , Alberto Fraile ${ }^{\text {a }}$, Teodor Huminiuc ${ }^{\text {b }}$, Huseyin Sener Sen ${ }^{\text {a }}$, Emilio Frutos a Tomas Polcar ${ }^{\mathrm{a}, \mathrm{b}^{*}}$

${ }^{\text {a }}$ Department of Control Engineering, Faculty of Electrical Engineering, Czech Technical University in Prague, Technicka 2, 16627 Prague 6, Czech Republic

${ }^{\mathrm{b}}$ Engineering Materials, Faculty of Engineering and Physical Sciences, University of Southampton, Southampton SO17 1BJ, UK

\begin{abstract}
:
Achieving the theoretical strength of a metallic alloy material is a demanding task that usually requires utilizing one or more of the well-established routes: i. decreasing the grain size to stop or slow down the dislocation mobility, ii. adding external barriers to dislocation pathways, iii. altering the crystal structure, or iv. combining two of the previous discrete strategies, i.e., implementing crystal seeds into an amorphous matrix. Each of the outlined methods has clear limitations, hence further improvements are required. We present a unique approach that envelops all the different strength-building strategies together with a new phenomenon - phase transition. We simulated the plastic deformation of a $\mathrm{Zr}-\mathrm{Nb}$ nanolayered alloy using molecular dynamics and ab initio methods, and observed the transition of $\mathrm{Zr}$ from HCP to FCC, and then to BCC during compression. The alloy, which was prepared by magnetron sputtering, exhibited near-theoretical hardness $(10.8 \mathrm{GPa})$ and the predicted transition of the $\mathrm{Zr}$ structure was confirmed. Therefore, we have identified a new route for improving the hardness of metallic alloys.
\end{abstract}

Keywords: nanolayered materials; metallic alloy; phase transition; interfaces; coating

Corresponding author: polcar@fel.cvut.cz

\section{Introduction:}

Increasing the strength of crystalline materials is one of the main driving purposes in the history of material research. To achieve this challenging goal, several microstructural design 
procedures were devised in order to approach the theoretical limits. After gaining a clearer understanding of dislocation phenomena that underpin plasticity, subsequent attempts focused on the most natural strategy: stopping or slowing down the dislocation mobility. Initial design attempts focused on adding more barriers to dislocation glide pathways, an effect commonly known as Hall-Petch strengthening. This cemented the inverse proportionality between the average grain size and the resulting strength of a polycrystalline material ${ }^{1}$. This was followed by the addition of extensive inner-boundaries resulting in twin boundary strengthening. ${ }^{2-9}$ Finally, a modern design for boundary-dominated strengthening has become prominent, achieved by stacking series of binary systems with defined periodicity yielding multilayered nano-composites $(\mathrm{MNCs})^{10-14}$. All these efforts were limited to a minimum crystallite size threshold of approximately $10 \mathrm{~nm}^{15}$ due to the inverse Hall-Petch effect. In connection to HallPetch strengthening, the second design approach is known as precipitation or solute-hardening. The alloying elements create additional entropy and barriers to the dislocation motion ${ }^{16,17}$, however, similarly to the previous approaches, the size threshold values come into play ${ }^{18-22}$. Metallic glasses are promising candidates among strength-inducing microstructures due to their unique structural capabilities. In contrast to the crystalline counterparts, metallic glasses have neither grain boundaries nor crystal structure; thus, the deformation can take place predominantly via the formation of shear bands. Thanks to the transformation from dislocation based plasticity to amorphous deformation, the strength of these metals doubles, and even higher values are reached in modern alloys combining dual-phase strengthening behaviour. However, this comes with an important drawback: the loss of ductility. Beyond $2 \%$ of plastic strain, metallic glasses show drastic failure. A recent study ${ }^{19}$ presented a promising way to cover the size and side effects of the metallic and amorphous alloys by sputtering two phases together, although the fabrication of such an alloy as a bulk material is an enormous challenge.

\section{Results and Discussion}


The present study further advances material strengthening mechanisms through the implementation of the kinetic control of microstructures, which is achieved by manipulating the activation of position-dependent deformation mechanisms within sputtered $\mathrm{Zr} / \mathrm{Nb}$ multilayers. Selective stacking of amorphous and crystalline layers may provide a combination of all major strengthening mechanisms described above, together with a new component of localized phase transformation.

Using classical MD we can explore various effects in the deformation mechanism of a multilayered system, where layers differ in crystal structure (BCC-Nb or $\mathrm{HCP}-\mathrm{Zr}$ ) as well as in crystallization state, from amorphous to perfect crystalline for both elements. Figure 1 presents three distinct phase transitions taking place during indentation: a) HCP to BCC (+FCC) phase transition in $\mathrm{Zr}$, b) amorphous to $\mathrm{HCP}$ (with small regions of $\mathrm{BCC}$ and $\mathrm{FCC}$ ) in amorphous $\mathrm{Zr}$ and c), similarly, amorphous to $\mathrm{BCC}$ in $\mathrm{Nb}$ amorphous layers. These phase transitions are crucial to understanding the superior hardness achieved in our system, and they have been indeed identified in our experiments. No phase transition is observed in $\mathrm{BCC} \mathrm{Nb}$ under indentation, only an obvious reorientation of the crystal structure around the indenter tip. In the case of bulk Zr, the BCC and FCC phases are stable only during indentation; they revert to HCP during unloading in the MD simulations. However, there is the possibility of a stable BCC $\mathrm{Zr}$ phase in a multilayer structure, which depends on the layer thicknesses as discussed by Thomson $^{23}$. A detailed account of the simulation settings is provided in the supporting information (SI) and in Figure S1. 

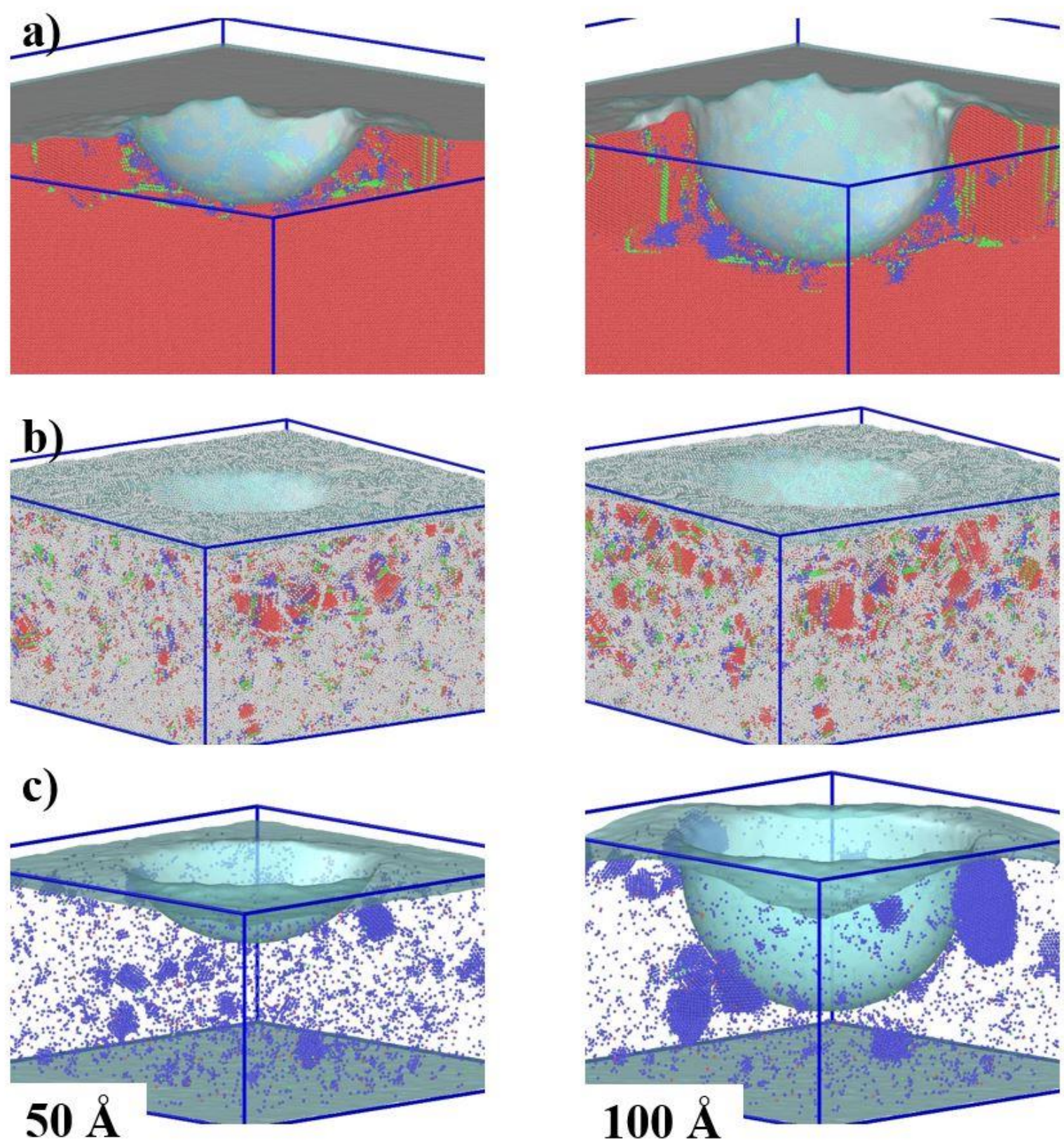

Figure 1. Phase transitions occurring during indentation. a) $\mathrm{HCP} \mathrm{Zr}$ (single crystal). b) $\mathrm{Zr}$, amorphous. c) $\mathrm{Nb}$, amorphous, penetration depth 50 and $100 \AA$ left and right, respectively. The colour code highlights the crystal structure: blue $=\mathrm{BCC}$, red $=\mathrm{HCP}$, green $=$ FCC ( (disordered atoms $($ white $)$ have been deleted in figures a) and $\mathrm{c})$ for clarity). The number in the bottom-left corner in each figure indicates the indentation depth.

To support our classical MD simulations and reveal the underlying mechanism pivotal for our design strategy, we performed a comprehensive ab initio study regarding the $\mathrm{Zr}$ phase transition under pressure and the effect of the $\mathrm{Nb}$ interface. Firstly, $\mathrm{HCP}-\mathrm{Zr}$ was compressed along the $\mathrm{c}$ 
axis (normal to the (0001) plane) by decreasing the value of the lattice constant in this direction. The phase transition is observed at about $40 \%$ compression at $0 \mathrm{~K}$. Figure 2 shows the energy profile for this compression process, setting the total energy in a fully relaxed state as a reference for both the $0 \mathrm{~K}$ and $300 \mathrm{~K}$ case. The results indicate that there is a $0.3 \mathrm{eV} /$ atom energy barrier for the HCP-BCC transition and the energy difference between these phases is about 0.13 $\mathrm{eV} /$ atom at $0 \mathrm{~K}^{24}$. At $300 \mathrm{~K}$ these values drop to $0.11 \mathrm{eV} /$ atom and $0.05 \mathrm{eV} /$ atom, respectively, in agreement with previous studies ${ }^{25}$. Moreover, an intermediate FCC phase is observed, in agreement with both our classical MD simulations and previous studies ${ }^{24,25}$, as shown in the inset of Figure 2. The drop in temperature decreases not only the energy barrier but also the compression required for the transition to occur. It is worth noting that the pressure applied during the simulations did not exceed 2.5 GPa. Hence, the ab-initio results demonstrate the possibility of the phase transformation in $\mathrm{Zr}$ from $\mathrm{HCP}$ to $\mathrm{BCC}$ by indentation normal to the (0001) plane. When the pressure on BCC-Zr is lifted, it transforms back to the original HCP structure, which corroborates our MD simulations. In the second step of the calculations, we created $\mathrm{BCC} / \mathrm{BCC}$ and $\mathrm{HCP} / \mathrm{BCC}-\mathrm{Zr} / \mathrm{Nb}$ multilayers in order to understand whether the $\mathrm{Nb}$ BCC interface can support $\mathrm{Zr}$ layers to retain the transformed BCC atomic arrangement. Since the emerging orientation in BCC-Zr post indentation is (100) we created a $(100) /(100)$ $\mathrm{BCC} / \mathrm{BCC} \mathrm{Zr} / \mathrm{Nb}$ interface. When we fully relaxed (both the atoms and lattice vectors) this system at $0 \mathrm{~K}$, the BCC/BCC structure with an in-plane lattice constant of $3.30 \AA$ was stable. Therefore, the $\mathrm{Nb}$ interface is vital to stabilize $\mathrm{BCC}-\mathrm{Zr}$ formed during plastic deformation. 


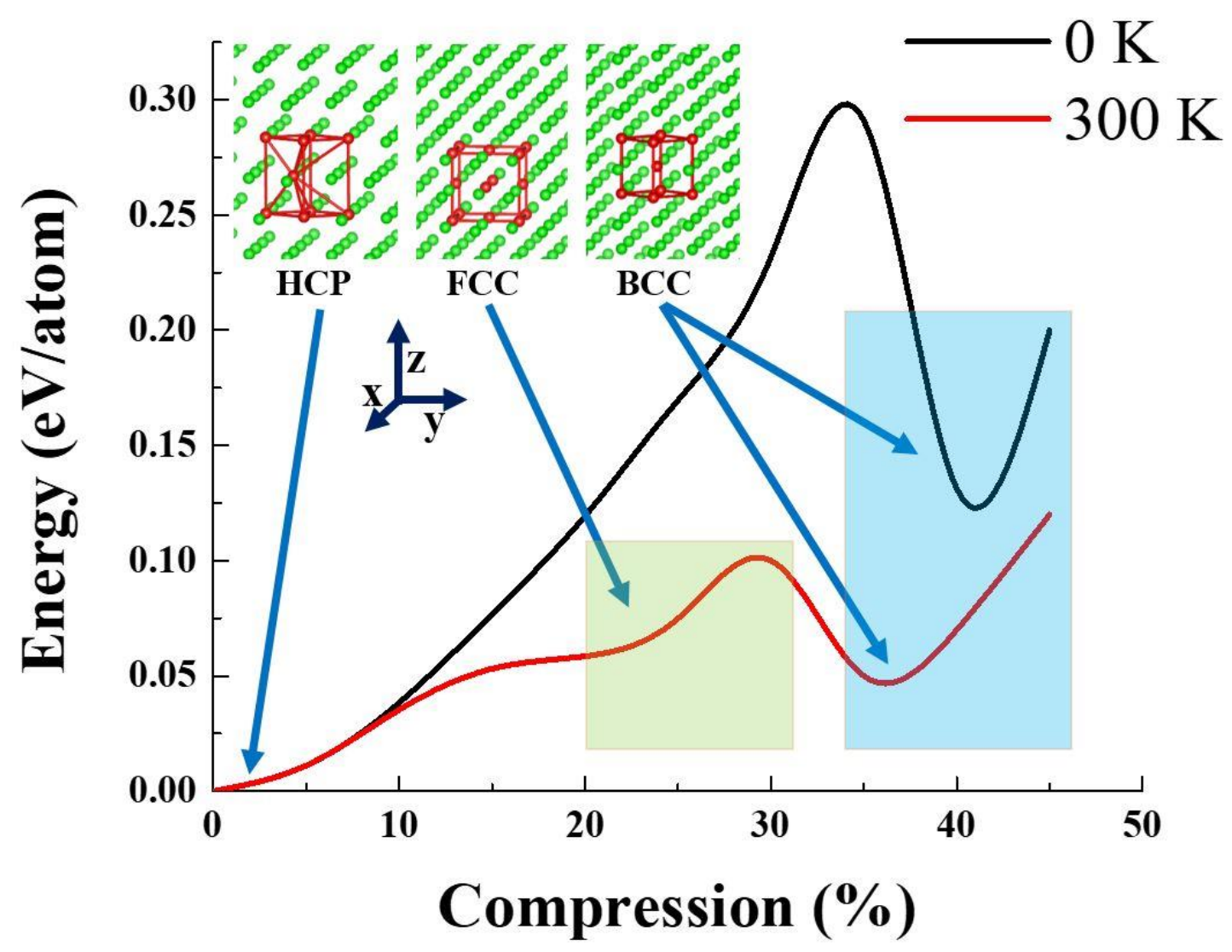

Figure 2. Energy change per atom for pressure-induced HCP to BCC transformation of zirconium at 0 and $300 \mathrm{~K}$. Coloured boxes indicate the intermediate FCC (green) and final BCC phase (blue). The region outside the boxes is HCP.

Depositing the selected amorphous/crystalline interfaces in the form of a nanolayered coating is a challenging endeavour and requires rigorous control of incident atom energy. During the sputtering process, due to the momentum transfer from one atom to another, the exhausted heat is transferred upwards (Figure 3c). Although the temperature difference between two separated layers is small, it is possible to create, gradually, crystal nuclei on top of each other. The results from our MD simulations show that the consumed kinetic energy leads to the formation of a heat-based crystal gradient between sputtered layers during the growth sequence, i.e., the activation of crystal seeds in the amorphous matrix is possible. This phenomenon is highlighted in Figure 3a, which shows the mechanism of thermal assistance in creating a gradual 
crystallization process. As the number of layers increases, the stored thermal energy overcomes the activation potential of the crystal phase. Within the initial layers, due to the lack of thermal energy, both $\mathrm{Zr}$ and $\mathrm{Nb}$ layers will be amorphous. After reaching a critical thickness and gaining sufficient thermal energy, nucleation will be attained firstly in the $\mathrm{Nb}$ layer (due to its higher symmetry), and then gradient crystallization will be observed in the following layers. Once the $\mathrm{Nb}$ layers become fully crystalline, $\mathrm{Zr}$ follows a similar trend, i.e., changing from completely amorphous to fully crystalline (see Figure 3a). In agreement with previous experimental and theoretical studies, our simulations indicate that the use of highly energetic atoms during thinfilm deposition promotes layer-by-layer growth (usually referred to as Frank-van-der-Merve growth $^{26}$ ) resulting in an amorphous metallic phase. At low incident-atom energies, threedimensional islands form on the surface and subsequently coalesce into a polycrystalline film (Volmer-Weber growth) ${ }^{27}$. Figure $3 \mathrm{~b}$ shows the difference between the pair distribution functions $(\mathrm{g}(\mathrm{r}))$ of $\mathrm{Zr}$ and $\mathrm{Nb}$ in pure crystalline and amorphous phases.
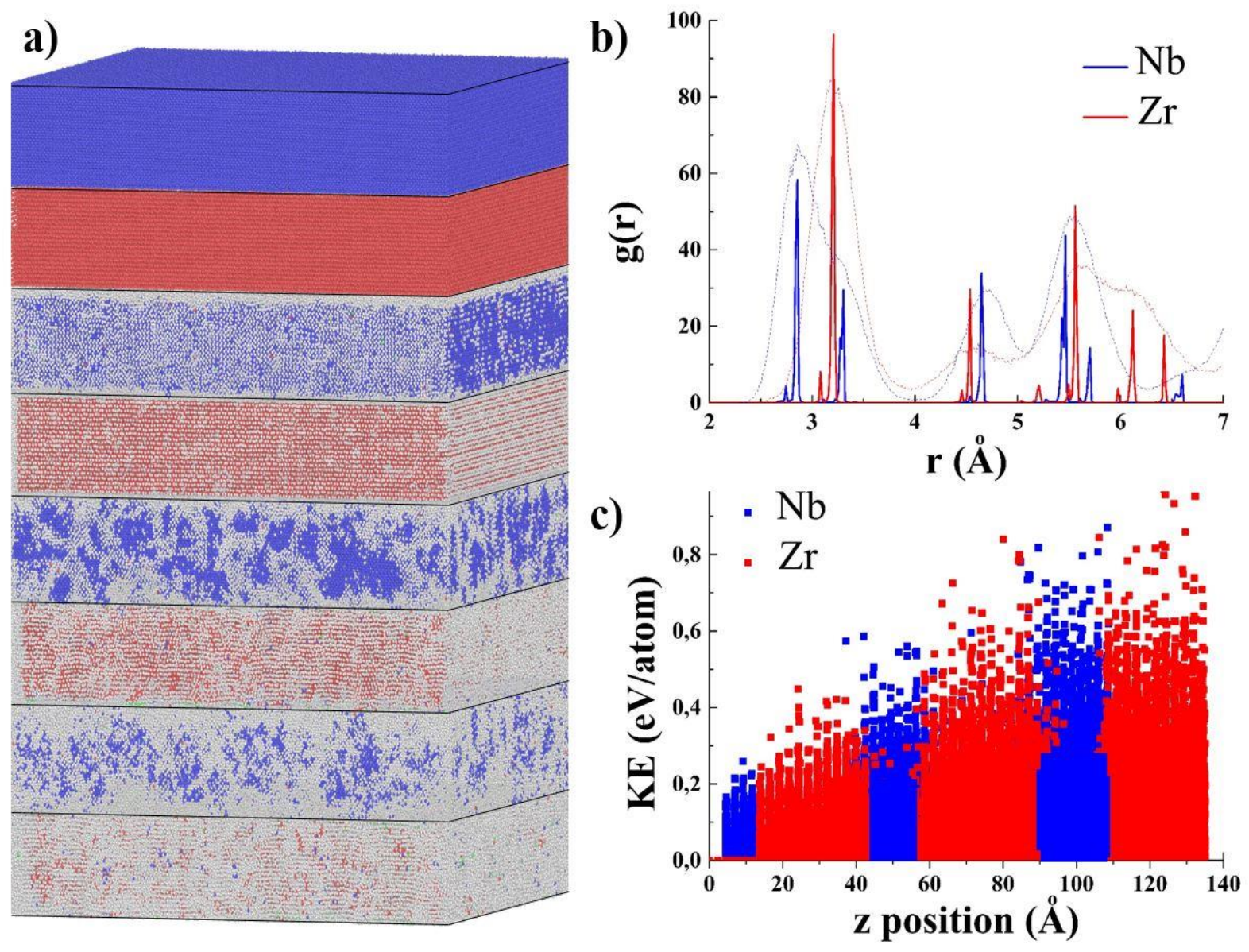
Figure 3. a) Simulated evolution of crystallinity in an alternating Nb-Zr layered system during coating growth. Colour code: blue $=\mathrm{BCC}(\mathrm{Nb})$, red $=\mathrm{HCP}(\mathrm{Zr})$, white $=$ disordered atoms. In our samples, the thickness of every layer is $6 \mathrm{~nm}$. b) Pair distribution functions, $\mathrm{g}(\mathrm{r})$, of $\mathrm{Zr}$ and $\mathrm{Nb}$ in pure crystalline and amorphous phases. c) Kinetic energy per atom of both $\mathrm{Nb}$ (blue) and $\mathrm{Zr}$ (red) during the deposition simulation.

Following the atomistic design perspectives, we realize our hypothesis with a multilayered $\mathrm{Zr}$ $\mathrm{Nb}$ model system deposited on [111] oriented Si substrates. The high kinetic energy of incident atoms required by our simulations was obtained by magnetron sputtering at low process pressure, thus increasing the mean free path of incident atoms. The layer-to-layer heat transfer was enhanced by high power to the metallic targets.

Figure 4 shows the resulting crystallinity gradient in the as-deposited $\mathrm{Zr}-\mathrm{Nb}$ multilayer. Roughly the first 10 layers are fully amorphous, followed by a combination of crystalline $\mathrm{Nb}$ and amorphous $\mathrm{Zr}$, and finally a fully crystalline bilayer. High resolution images show the interfaces in the crystalline upper part of the coating. The Nb layers are usually oriented with the [220] and [100] family of planes in the growth direction, which points to a $45^{\circ}$ rotation of the unit cell relative to the normal position on the [111] and [001] zone axes. Neighbouring $\mathrm{Nb}$ grains show well-defined coherent interfaces with no significant defect density. Along the same layer, multiple rotations between the two-zone axis are noted. The lateral grain size of $\mathrm{Nb}$ ranges from 5 to $10 \mathrm{~nm}$. The HCP $\mathrm{Zr}$ interlayer is mostly oriented with the basal plane (0001) in the plane of observation and bridges both the [001] and [111] oriented $\mathrm{Nb}$ grains. The in-plane orientation of $\mathrm{HCP} \mathrm{Zr}$ changes less often when compared to $\mathrm{Nb}$; however, the general trend is for the unit cell to lay with the long axis in the plane. It is worth noting that the number of defects is much higher at the $\mathrm{Nb}[001] / \mathrm{Zr}[0001]$ interface due to higher lattice mismatch (see SI, Figure S3). 

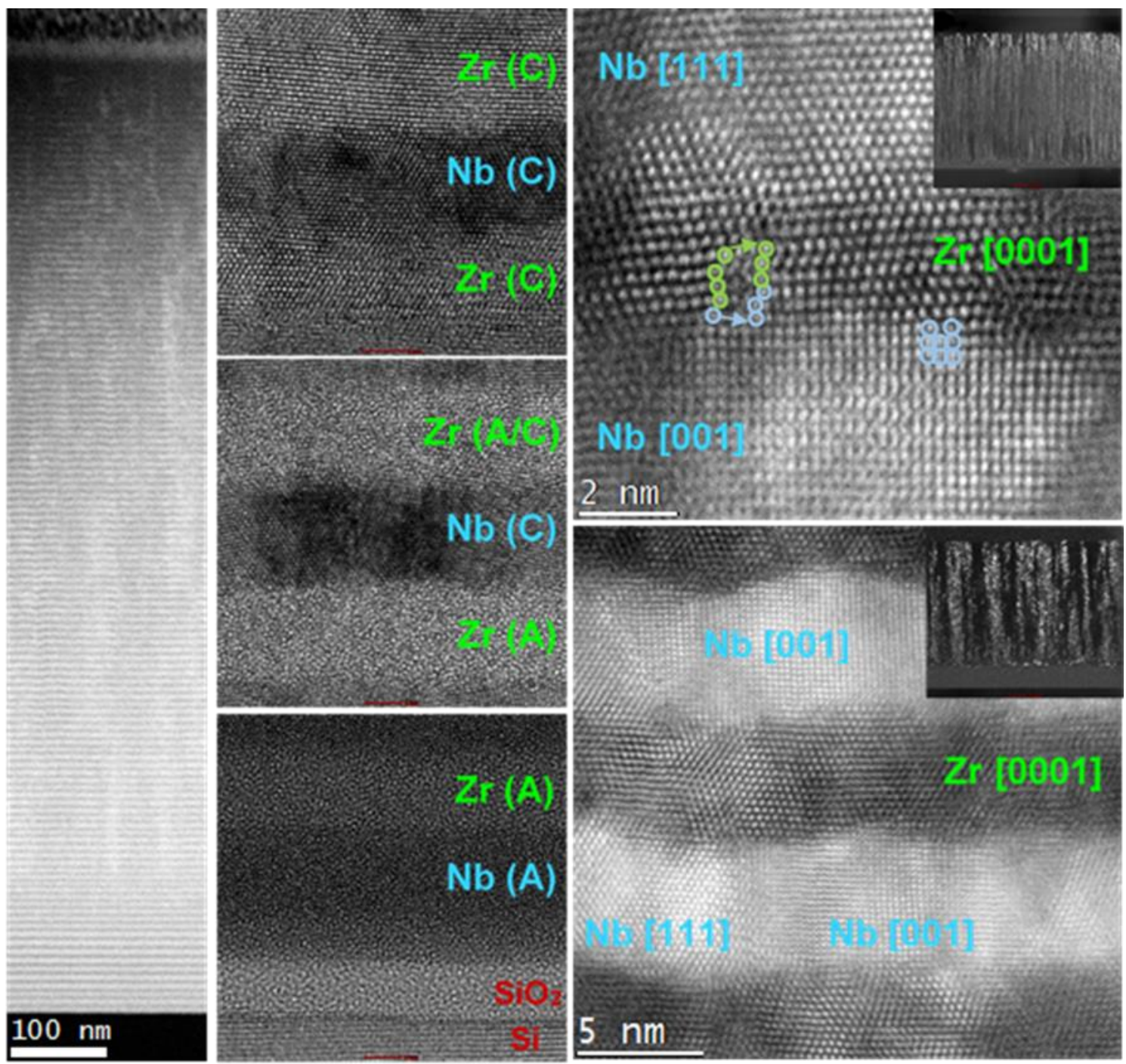

Figure 4: HAADF images of as-deposited $\mathrm{Zr} / \mathrm{Nb}$ multilayer (left) with amorphous layers at the bottom, transition region between amorphous and crystalline parts in the center and fully crystalline layers at the upper part of the coating (see figures on the right for detail).

Finally, we carried out multiple load nanoindentation tests to investigate the mechanical response of our deformation-controlled-design (DCD). The measured hardness was $10.8 \mathrm{GPa}$, significantly higher than that of a fully crystalline $\mathrm{Zr} / \mathrm{Nb}$ multilayer $(4.8 \mathrm{GPa})^{28}$ and almost reaching the theoretical threshold value of the $\mathrm{Zr}-\mathrm{Nb}$ nanocomposite system. 
Detailed investigation of loading curves is a suitable tool to study the activation of slip and twinning systems $s^{2-5}$ as a function of nanolayer composition ${ }^{29,30}$, and/or as a function of crystalline/amorphous layer combination ${ }^{31-33}$. A simple approach to study the activation of different slip systems in each nanolayer is through small variations, known as 'pop-in', recorded along the loading curve of a nanoindentation test (Figure S2). From the load derivative curve $(d P / d h$ vs. the indentation depth, $h)$ it is possible to identify the exact position of the first popin representing the plasticity onset. Then we can calculate the maximum resolved shear stress, $\tau_{R S S}$, for the plasticity initiation, which is $7.3 \mathrm{GPa}$ (for more details see $\mathrm{SI}$ ). This value is unusually high, since for metallic materials, $\tau_{R S S}$ should be between $G / 15$ and $G / 30$, where $G$ is the shear modulus calculated as $G=E /(2(1+v))$, and $v$ is the dimensionless Poisson coefficient. In fact, the $\tau_{R S S}$ of the $\mathrm{Zr} / \mathrm{Nb}$ nano-multilayer is quite close to the theoretical maximum resolved shear stress value, $\tau_{T R S S}=G / 2 \pi(8.5 \mathrm{GPa})$, shown by a material without dislocation activity. The proximity of $\tau_{R S S}$ and $\tau_{T R S S}$ values suggests that the plastic deformation of the $\mathrm{Zr} / \mathrm{Nb}$ nano-multilayer system takes place with few dislocations.

An important indication of the possible strengthening mechanism is a large difference between the geometrically necessary dislocation density, $\rho_{\text {gnd }}$, which is generated in a small and confined plastic deformation volume, and the stored dislocation density $\rho_{s d d}$ (see SI).

The generation of dislocations and their evolution in this system is obviously very complex and further analysis by molecular dynamics is presented in the supporting information. $\mathrm{Zr}$ undergoes a complicated HCP to BCC phase transition through a metastable FCC (See Movie 3), which cannot be followed experimentally with ex-situ observation.

The effects of the plastic deformation on the microstructure of the multilayer are shown in Figure 5, where the stress applied through the indenter triggers the crystallisation of the amorphous lower region of the coating. The low magnification images show the protrusion of the crystalline phase into the glass-like layers that follow the contour of the indenter tip. The 
high and localized compressive stress causes the formation of crystallites $40 \mathrm{~nm}$ closer to the substrate interface in the amorphous region, thus confirming a different energy dissipation mechanism: crystallization of the glassy phase under compression. At the nanoscale, the compression exerted on the multilayer system causes the local phase transformation of $\mathrm{Zr}$ from $\mathrm{HCP}$ to BCC. Significant thinning of the $\mathrm{Zr}$ layer down to $2 \mathrm{~nm}$ is observed, which is attributed to both plastic flow and the change of atomic order. The phase transition is usually observed when the $\mathrm{Zr}$ is sandwiched between two Nb layers with the same [100] orientation, forming an almost perfect epitaxial interface between $\mathrm{Nb}[100]-\mathrm{Zr}[100]-\mathrm{Nb}[100]$. Another type of stacking where the dominant direction becomes [111] is presented in the supporting information (Figure S4 and Figure S5). The stress-dependent transformation is proven by the occurrence of partial transformation around the indented regions where both the HCP and BCC phases of Zr coexist (see SI). As suggested by our DFT simulations, the close lattice match of the two cubic phases plays an important role in the structural transformation and stability of BCC Zr.

Our MD and ab initio simulations successfully predicted the phase transition of the Zr layer during nanoindentation, which was observed in experiment. Furthermore, our ab initio study confirmed that a thin BCC $\mathrm{Zr}$ layer is stabilized by BCC Nb. Nevertheless, there is still a long way to rely purely on the computational design of metallic nanolayered alloys. Some limitations, such as the small size of the system (up to a few million atoms), and simulation times (ns), are well known and their effect can be estimated. However, there are only a few suitable force fields for any metal combination that can be applied in MD simulations of the indentation of the layered system; in our case, some of the existing $\mathrm{Zr}-\mathrm{Nb}$ potentials seem unsuitable and likely could be improved. Our ab initio study of the $\mathrm{Zr}$ phase transition uses perfect crystals; studying the effect of all possible defects (e.g., vacancies, dislocations) is not possible with existing computing power. We can thus simulate only a fraction of possible events during the plastic deformation of nanolayers. To confirm that simulations unravel key 
phenomena controlling the deformation process, experimental verification is still a vital element in material design.
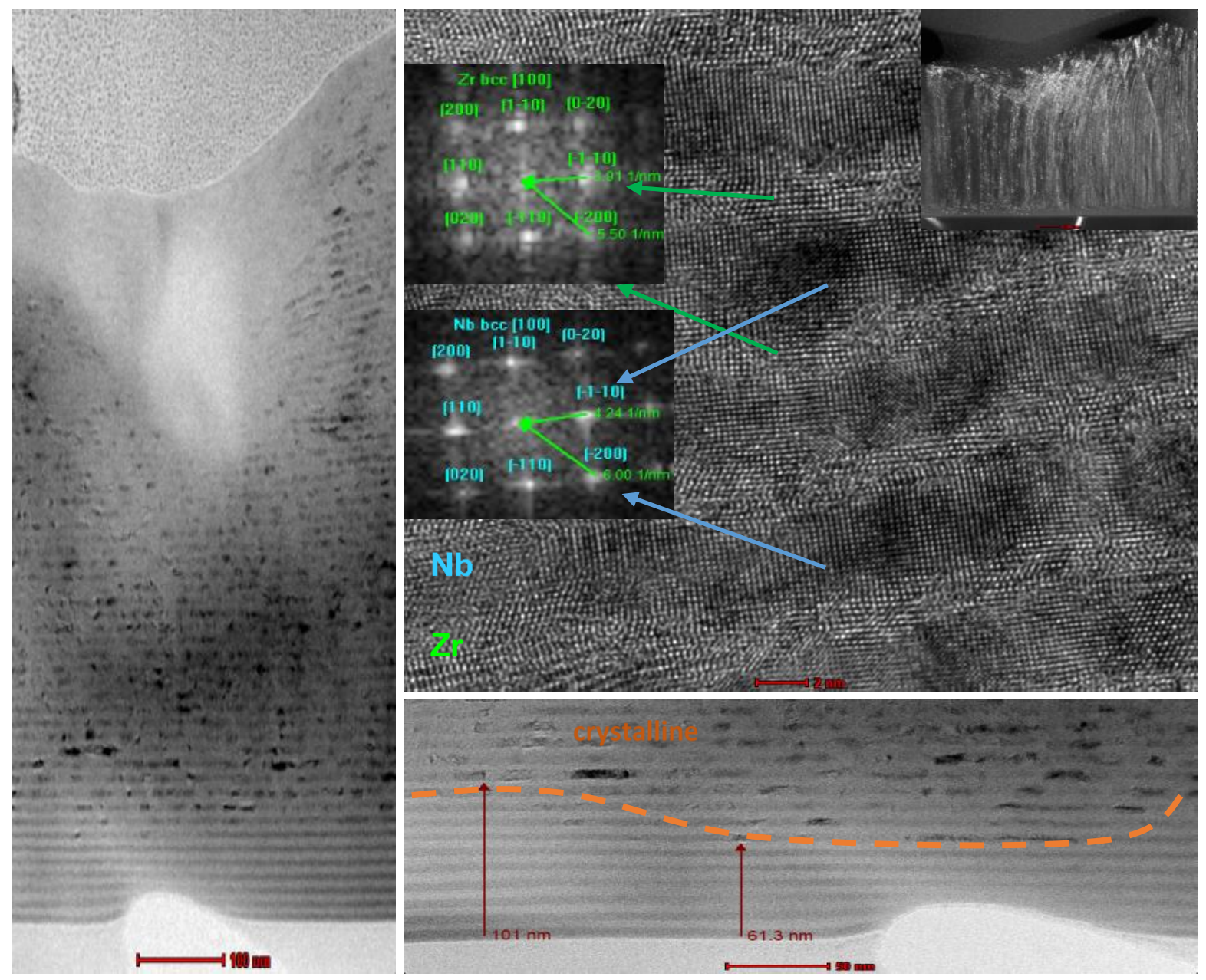

Figure 5: HR TEM images of deformed $\mathrm{Zr} / \mathrm{Nb}$ multilayer (left) highlighting the stress-induced crystallisation and $\mathrm{HCP}$ to BCC phase transformation of $\mathrm{Zr}$ following plastic deformation (right).

\section{Conclusions}

We designed a multilayered-metallic nano-composite material in which multiple deformation modes are activated separately by the local arrangement of crystal structure during single step magnetron sputtering. The major goal here is adding another milestone to the historical material strengthening race by controlling dominant deformation mechanisms within the confined 
volume of $\mathrm{Zr}-\mathrm{Nb}$ nanocomposites. The key idea is to consume additional deformation energy by confined space phase transformation and glass-like phase crystallisation.

The starting hypothesis is supported by advanced experimental and nanoscale computational studies, and results in a significant strengthening of the $\mathrm{Zr}-\mathrm{Nb}$ multilayer with a hardness of $10.8 \mathrm{GPa}$, which is approximately $6 \mathrm{GPa}$ higher than its homogenous crystalline counterparts. Moreover, there is plenty of room for improvement by optimizing layer thicknesses and/or a combination of amorphous and crystalline layers. The concept applies to various combinations of metals and represents a promising way to produce strength-tunable alloys for extreme engineering applications.

\section{Supporting Information}

Classical MD methods, Figure S1, Ab initio calculations, Table S1, Experimental details, Figure S2, Figure S3, Figure S4, Figure S5, Movie 1, Movie 2, Movies 3, References.

\section{Acknowledgments}

This work was supported by the project GACR 17-17921S and by OPVVV grant Novel nanostructures for engineering applications No. CZ.02.1.01/0.0/0.0/16_026/0008396. Our MD and ab initio simulations were carried out in Anselm \& Salomon supercomputers, IT4I, Ostrava, Czech Republic, and supported by The Ministry of Education, Youth and Sports from the Large Infrastructures for Research, Experimental Development and Innovations project “IT4Innovations National Supercomputing Center - LM2015070”. The electron imaging was performed with the support of the South of England Analytical Electron Microscope (EP/K040375/1), within the David Cockayne Centre for Electron Microscopy, Department of Materials, University of Oxford, and with the support of CEITEC Nano Research Infrastructure 
(ID LM2015041, MEYS CR, 2016-2019), CEITEC Brno University of Technology. An insightful discussion with Paolo Nicolini and Benjamin Irving is greatly acknowledged.

\section{Author contributions}

H.Y., A.F. and T.P. designed experiments; A.F. and H.S. performed MD and ab initio simulations, H.Y. produced samples, H.Y. and T.H. performed nanoindentation, E.F. analyzed the indentation data, T.H. carried out TEM analysis, T.P. summarized and critically assessed all data. All authors contributed to the writing of the manuscript.

\section{Conflicts of Interest}

The authors declare no conflict of interest.

\section{References}

(1) Merz, M. D.; Dahlgren, S. D. Tensile Strength and Work Hardening of Ultrafine-Grained High-Purity Copper. J. Appl. Phys. 1975, 46, 3235-3237.

(2) Kulkarni, Y.; Asaro, R. J. Are some Nanotwinned FCC Metals Optimal for Strength, Ductility and Grain Stability? Acta Mater. 2009, 57, 4835-4844.

(3) Lu, L.; Zhu, T.; Shen, Y.; Dao, M.; Lu, K.; Suresh, S. Stress Relaxation and the Structure Size-Dependence of Plastic Deformation in Nanotwinned Copper. Acta Mater. 2009, 57, $5165-5173$.

(4) Wang, G.; Li, Guangyu; Zhao, L.; Lian, J.; Jiang, Z.; Jiang, Q. The Origin of the Ultrahigh Strength and good Ductility in Nanotwinned Copper. Mater. Sci. Eng. A 2010, $527,4270-4274$

(5) Zhao, Y.; Furnish, T. A.; Kassner, M. E; Hodge, A. M. Thermal Stability of Highly Nanotwinned Copper: The Role of Grain Boundaries and Texture. J. Mater. Res. 2012, 27, 3049-3057. 
(6) Lu, L.; Chen, X.; Huang, X.; Lu, K. Revealing the Maximum Strength in Nanotwinned Copper. Science 2009, 323, 607-610.

(7) Bufford, D.; Liu, Y.; Wang, J.; Wang, H; Zhang, X. In situ Nanoindentation Study on Plasticity and Work Hardening in Aluminium with Incoherent Twin Boundaries. Nat. Commun. 2014, 5, 4864.

(8) Jang, D.; Li, X.; Gao, H; Greer, J. R. Deformation Mechanisms in Nanotwinned Metal Nanopillars. Nat. Nanotechnol. 2012, 7, 594-601.

(9) Zhang, X.; Misra, A.; Wang, H.; Nastasi, M.; Embury, J. D.; Mitchell, T. E.; Hoagland, R. G.; Hirth, Nanoscale-Twinning-Induced Strengthening in Austenitic Stainless Steel Thin Films. J. P. Appl. Phys. Lett. 2004, 84, 1096-1098.

(10) Callisti, M.; Polcar, T. Combined Size and Texture-Dependent Deformation and Strengthening Mechanisms in Zr/Nb Nano-multilayers. Acta Mater. 2017, 124, 247-260.

(11) Frutos, E.; Callisti, M.; Karlik, M.; Polcar, T. Length-Scale-Dependent Mechanical Behaviour of $\mathrm{Zr} / \mathrm{Nb}$ Multilayers as a Function of Individual Layer Thickness. Mater. Sci. Eng. A 2015, 632, 137-146.

(12) Callisti, M.; Karlik, M.; Polcar, T. Bubbles Formation in Helium Ion Irradiated Cu/W Multilayer Nanocomposites: Effects on structure and mechanical properties. J. Nucl. Mater. 2016, 473, 18-27.

(13) Qin, E. W.; Lu, L.; Tao, N. R.; Lu, K. Enhanced Fracture Toughness of Bulk Nanocrystalline Cu with Embedded Nanoscale Twins. Scr. Mater. 2009, 60, 539-542.

(14) Sun, B. B.; Sui, M.L.; Wang, Y.M.; He, G.; Eckert, J.; Ma, E. Ultrafine Composite Microstructure in a Bulk Ti Alloy for High Strength, Strain Hardening and Tensile Ductility. Acta Mater. 2006, 54, 1349-1357.

(15) Yamakov, V.; Wolf, D.; Phillpot, S. R.; Mukherjee, A. K.; Gleiter, H. Deformation Mechanism Crossover and Mechanical Behaviour in Nanocrystalline Materials. Philos. Mag. Lett. 2003, 83, 385-393. 
(16) Salomons, E. HCP-BCC Transition and the Free Energies of the HCP and BCC Structures of Zirconium. Phys. Rev. B 1991, 43, 6167-6169.

(17) Wu, Z.; Bei, H.; Pharr, G. M.; George, E. P. Temperature Dependence of the Mechanical Properties of Equiatomic Solid Solution Alloys with Face-Centered Cubic Crystal Structures. Acta Materialia. 2014, 81, 428-441.

(18) Kumar, G.; Tang, H. X.; Schroers, J. Nanomoulding with Amorphous Metals. Nature 2009, 457, 868-872.

(19) Wu, G.; Chan, K. C.; Zhu, L.; Sun, L; Lu, J. Dual-phase Nanostructuring as a Route to High-Strength Magnesium Alloys. Nature 2017, 545, 80-83.

(20) Chou, H. S.; Du, X. H.; Huang, J. C.; Nieh, T. G. Time-Dependent Mechanical Behavior of Phase-Separated ZrCuTiTa Thin Films under Nanoindentation. Intermetallics 2012, 27, 26-30.

(21) Guo, H.; Yan, P. F.; Wang, Y. B.; Tan, J.; Zhang, Z. F.; Sui, M. L.; Ma, E. Tensile Ductility and Necking of Metallic Glass. Nat. Mater. 2007, 6, 735-739.

(22) Zheng, Q.; Cheng, S.; Strader; J. H.; Ma, E.; Xu, J. Critical Size and Strength of the Best Bulk Metallic Glass Former in the Mg-Cu-Gd Ternary System. Scr. Mater. 2007, 56, 161-164.

(23) Thompson, G.B.; Banerjee, R.; Dregia, S.A.; Fraser, H.L. Phase Stability of bcc Zr in Nb/Zr Thin Film Multilayers. Acta Mater. 2003, 51 5285-5294

(24) Dolgusheva, E. B.; Trubitsin, V. Y. Study of Size Effects in Structural Transformations of BCC Zr Films by Molecular-Dynamics Simulation. Comput. Mater. Sci. 2014, 84, 23 30.

(25) Peng, Q.; Wu, J.; Zheng, M.; Chen, H.; Tao, X.; Du, Y.; Peng, Q. An Interatomic Potential for Simulation of Defects and Phase Change of Zirconium. Comput. Mater. Sci. 2018, 147, 7-17.

(26) Frank, F. C.; Merwe, J. H. Van Der. One-Dimensional Dislocations. I. Static Theory. 
Proc. R. Soc. London. Ser. A. Math. Phys. Sci. 1949, 198, 205-216.

(27) Volmer, M.; Weber, A. Keimbildung in übersättigten Gebilden. Zeitschrift für Phys. Chemie 1926, 119U, 277-301.

(28) Callisti, M.; Polcar, T. Combined Size and Texture-Dependent Deformation and Strengthening Mechanisms in Zr/Nb nano-multilayers. Acta Mater. 2017, 124 247-260

(29) Monclús, M.; Karlik, M.; Callisti, M.; Frutos, E.; Lorca, J. L.; Polcar, T.; MolinaAldareguía, J.M. Microstructure and Mechanical properties of Physical Vapor Deposited $\mathrm{Cu} / \mathrm{W}$ Nanoscale Multilayers: Influence of Layer Thickness and Temperature. Thin Solid Films 2014, 571, 275-282

(30) Banerjee, S.; Robi, P. S.; Srinivasan, A.; Kumar, L. P. High Temperature Deformation Behavior of Al-Cu-Mg Alloys Micro-Alloyed with Sn. Mater. Sci. Eng. A 2010, 527, 2498-2503.

(31) Greer, A. L. Metallic Glasses. Science 267, 1947-1953 (1995). DOI: 10.1126/science.267.5206.1947

(32) Zhang, J.Y.; Liu, Y.; Chen, J.; Chen, Y.; Liu, G.; Zhang, X.; Sun, J. Mechanical Properties of Crystalline $\mathrm{Cu} / \mathrm{Zr}$ and Crystal-Amorphous $\mathrm{Cu} / \mathrm{Cu}-\mathrm{Zr}$ Multilayers. Mater Sci Eng A. 2012, 30 392-398.

(33) Fan, Z.; Xue, S.; Wang, J.; Yu, K. Y.; Wang, H.; Zhang, X. Unusual Size Dependent Strengthening Mechanisms of $\mathrm{Cu}$ /Amorphous CuNb Multilayers. Acta Mater. 2016, 120 327-336. 